\title{
Influence of the "Active Mix" supplement with separate administration of antagonistic components on the structure of the mice internal organs
}

\author{
D.S. Berestov*, Yu.G. Vasiliev, A.N. Kulikov, and N.V. Isupova \\ FSBEI of HE Izhevsk SAA, Izhevsk, Russia
}

\begin{abstract}
The question of the need for separate administration of feed additives' components that are antagonistic from the point of view of their chemical properties and physiological effects has long been debated among researchers. The authors have proposed a new supplement based on chelate compounds, which components' effect on the organism of laboratory animals has been partially considered earlier. However, the assessment of the components' effects on the morphology of the excretory and reproductive systems' organs has not been studied. In this regard, the work considered the effect of feed additive components with separate introduction of chemically and physiologically antagonistic components on the pathoanatomical picture, as well as the microstructure of the kidneys and testes of laboratory mice to assess potentially negative effects on the excretory and reproductive systems. The supplement was administered orally at various dosages. The organ structure was assessed after 1,2 weeks and 1 month. The microstructure of the kidneys and testes was histologically studied in the experiment and control. The negative influence absence of the supplement components in the entire range of applied dosages is shown. The absence of visible deviations of the pathoanatomical picture during autopsy of experimental animals was noted.
\end{abstract}

\section{Introduction}

High productivity of modern animals requires replenishment of intensive metabolism needs with feed additives. They are constantly being improved, new approaches are proposed. The expediency of maintaining trace elements in organic, chelated form has been shown $[3,5,6]$. This makes it possible to reduce the dosage of the introduced components without negatively affecting the health and productivity of animals [15], which is important from the point of view of the supplements' ingestion into products and, ultimately, into the human diet [11]. In addition, a number of authors suggest the need for separate introduction of feed additives' components in connection with their possible chemical $[7,10,16]$ and physiological [14] antagonism. There are different points of view on this problem. Some researchers believe that this approach does not justify itself, while others, on the contrary, give arguments in

\footnotetext{
* Corresponding author: berestovds@rambler.ru
} 
favor of the need to separate the components. However, research is underway and experimental results show the possibility to use such additives. However, potentially negative effects may not manifest themselves at the macrolevel, being revealed during the analysis of animals' internal organs microorganization, including due to the accumulation of additives' chemical substances [13]. There are very few works in the available literature that histologically evaluate the potentially negative effects of additive components. In most cases, the authors analyze the effect on productivity and the elements' content in the blood and various organs [8]. This determines the relevance of this work. As with the creation of any new product in such studies, it becomes necessary to assess both its positive and potentially negative effects on laboratory animals before carrying out production experiments on livestock in farm conditions, which determines the goals and objectives of this work.

\section{Aim and tasks}

The aim of the study was to assess the internal organs' microorganization of model animals against the introduction background of various "Active Mix" supplement doses with separate administration of the components. Considering the fact that the influence of the product components on the microorganization of the stomach, intestines, liver, trace elements content in blood, the behavior of animals was considered earlier [1, 2, 9], the tasks were to assess the pathoanatomical picture, the histological structure of the kidneys and testes.

\section{Materials and methods}

The studies were carried out on viripotent white male mice; they were kept in the vivarium of the Faculty of Veterinary Medicine of the Izhevsk State Agricultural Academy in accordance with the requirements generally accepted for this animal species. The mice were divided into 5 experimental and one control groups. The animals of the experimental groups received liquid supplement daily. The dietary supplement included fat-soluble vitamins (A, $\mathrm{D}, \mathrm{E}$ ), diacetophenonyl selenide emulsion, chelate complexes of manganese, iron, cobalt and zinc. Each mixture was administered orally every other day throughout the experiment. Thus, only one type of mixture was administered daily to exclude mixing of the antagonistic components. The supplement was administered to the animals per os through a dispenser for precise volume control. In this case, the mice swallowed the mixture on their own due to the peculiarities of its taste characteristics, which made it possible to exclude the use of a gastric tube and minimize the traumatic effect. Control group animals received an equivalent amount of normal saline. The distribution of animals into groups and the studied dosages are shown in Table 1.

Table 1. The number of animals in the experiment and their distribution into groups.

\begin{tabular}{|c|c|c|c|c|c|c|}
\hline \multirow[b]{2}{*}{ Study period } & \multicolumn{6}{|c|}{ Group name and dosage } \\
\hline & $\begin{array}{c}\text { No. } 1 \\
\text { additive, } \\
0,06 \\
\mathrm{~g} / \mathrm{kg}\end{array}$ & $\begin{array}{c}\text { No. } 2 \\
\text { additive, } \\
0,12 \\
\text { g/kg }\end{array}$ & $\begin{array}{c}\text { No. } 3 \\
\text { additive, } \\
0,3 \\
\mathrm{~g} / \mathrm{kg}\end{array}$ & $\begin{array}{c}\text { No. } 4 \\
\text { additive, } \\
0,6 \\
\text { g/kg }\end{array}$ & $\begin{array}{c}\text { No. } 5 \\
\text { additive, } \\
1,2 \\
\mathrm{~g} / \mathrm{kg}\end{array}$ & $\begin{array}{c}\text { control, } \\
\text { normal saline }\end{array}$ \\
\hline 1 week & 5 & 5 & 5 & 5 & 5 & 4 \\
\hline 2 weeks & 5 & 5 & 5 & 5 & 5 & 4 \\
\hline 1 month & 5 & 5 & 5 & 5 & 5 & 4 \\
\hline
\end{tabular}

The animals were withdrawn from the experiment after 1, 2 weeks and 1 month from its beginning. The killing was carried out by decapitation under general anesthesia. At autopsy, the pathoanatomical picture was visually assessed and the kidneys and testes were sampled 
into neutral buffered formalin for histological examination, which was carried out by conventional methods with the preparation of paraffin sections with further staining with hematoxylin-eosin and descriptive analysis under a microscope. The study was carried out in full at the Department of Anatomy and Physiology of the FSBEI HE Izhevsk SAA.

\section{Research results}

1 week from the start of the experiment. In the kidneys' histological preparations of the control, the cortex and marrow were clearly separated, the tubular apparatus was preserved (Fig. $1 \mathrm{~A}$ ), the proximal and distal tubules were well differentiated, their epithelium had visible basal striation. The connective tissue layers are thin, with few fibroblastic and monocytic-macrophagic row cells. In some parts of the organ, the number of dilated blood capillaries was slightly increased. The structure of the vascular glomeruli is typical. Glomerular blood supply is moderate, the lumen of the renal corpuscles capsules is moderately expanded. The marrow was composed of the epithelium of thin, distal straight tubules, collecting tubes. The epithelium of the tubules was typical, not changed. The transitional epithelium of the only papilla and pelvis of the organ was composed of 2-3 layers of cells. The connective tissue environment contained few diffusely distributed cells.

Samples of the adrenal glands were found near some of the kidney sections. Their structure was typical. In the glomerular layer, the cells differed in that the lipid inclusions in the cytoplasm were small. The corticocytes of this layer formed short glomerular structures. Connective tissue layers were thin. Separate figures of mitosis were identified in the primordial layer composed of small cells arranged in 3-5 rows. Light spongiocytes predominated in the bundle layer of the cortex. Dark spongiocytes were sporadic. The reticular zone was poorly developed. The marrow organization was typical. A significant amount of dust-like granules was found in norepinephrocytes and epinephrocytes.

The testes had a typical structure; the undulation of the spermatogenesis process was observed in the segments of longitudinal sections of the convoluted tubules with alternating zones of mature spermatozoa and cells of the spermatogenic series. The lumens of the convoluted tubules and the cavity of the appendage canal were filled with sperm.

In experimental group 1, the kidneys had a typical structure (Fig. 1 B). One specimen showed traits of venous stasis in the cortex and increased blood filling of the capillary bed, which was most likely a consequence of thanatogenesis. The structure of the testes did not differ from that in the control group.

In the 2nd experimental group, the histological picture of the kidneys (Fig. $1 \mathrm{C}$ ) and testes did not differ from the control.

In the 3rd experimental group in the kidneys' sections in the group as a whole, the picture corresponded to the control preparations (Fig. 1 D). One sample showed traits of a slight increase in the cortex blood supply, flattening of epithelial cells, both proximal and distal tubules, with a moderate expansion of their visible lumen (but not the total diameter of the tubules). The structure of the testes did not reveal any significant differences from the control.

In the 4th experimental group, no pronounced differences from the control were found in the kidney preparations (Fig. 1 E). In two samples, an increased blood filling of the venous vessels was found, which was a consequence of the dying process. In the testes preparations, the picture corresponded to the control. In one sample, the number of spermatozoa in the appendage canal was slightly increased in comparison with other animals of the group.

In the 5th experimental group in the kidneys' preparations, the picture corresponded to the control (Fig. 1 F). One sample showed minor traits of venous stasis. The microscopic picture of the testes did not reveal any differences from the control group. 

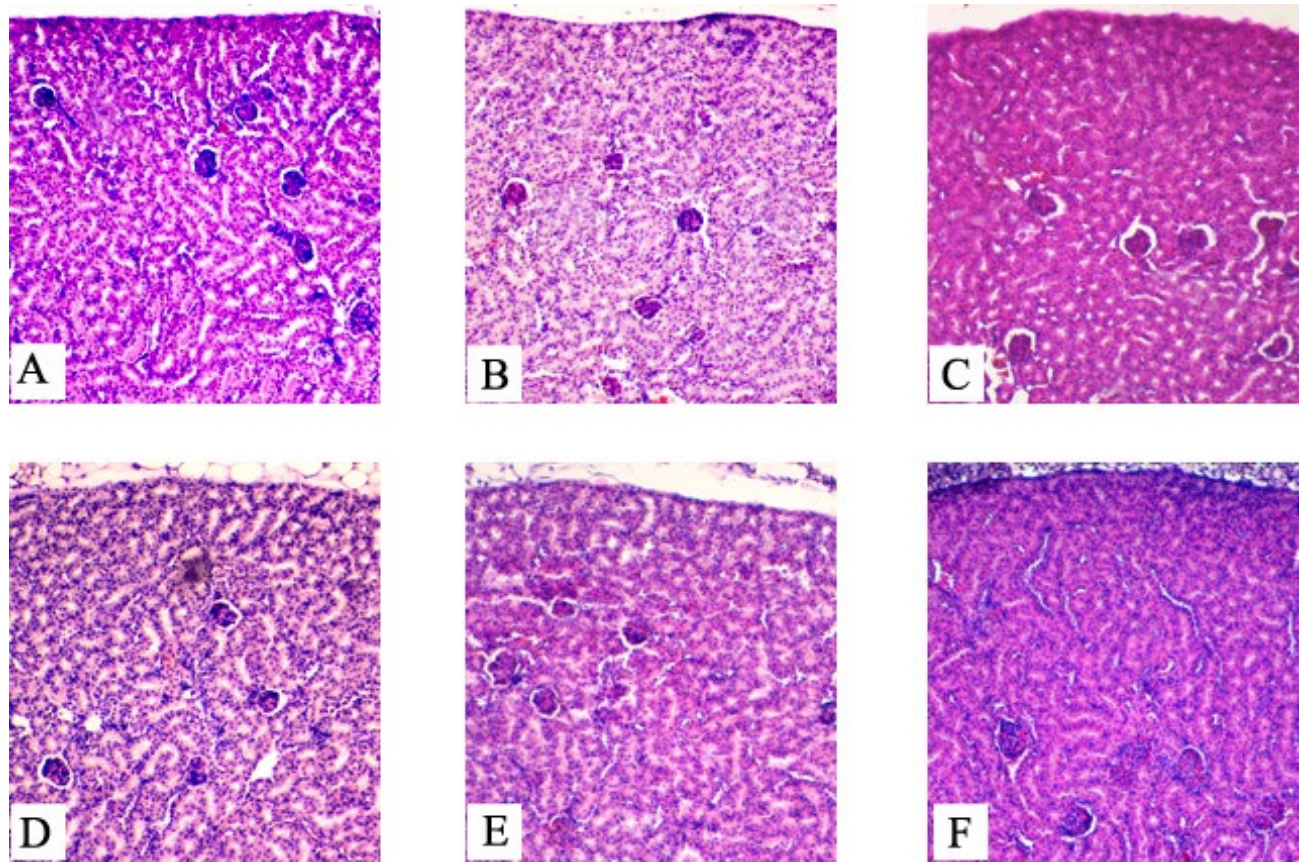

Fig. 1. Histological picture of the kidneys after 1 week from the beginning of the experiment. Average magnification: A - control, B - 1st experimental group, C - 2nd experimental group, D - 3rd experimental group, E - 4th experimental group, F - 5th experimental group. Explanations in the text.

Pathoanatomical picture after 1 week. Internal examination of the control group mice established the anatomically correct position of the internal organs. Serous membranes were shiny, moist. The omentum and mesentery contained a small amount of adipose tissue. In the stomachs - a moderate amount of gray contents forming a fine flocculent suspension (food residues) when mixed with water. The contents of the small intestine were gray-yellow in color and of mushy consistency. The distal large intestine contained segmented feces (boles) of solid consistency and dark color, typical for this animal species. The liver and kidneys were normal. There was a moderate amount of adipose tissue around the kidneys. In the lungs, areas of bright red color with a transverse size of up to $2 \mathrm{~mm}$ were detected - a consequence of hemaspiration during decapitation.

Autopsy of the animals treated with supplements did not reveal any visible macroscopic differences from the control.

2 weeks from the start of the experiment. In the control samples of all organs two weeks after the beginning of the experiment, the same picture was observed as in the control group of the previous period (Fig. 2). 

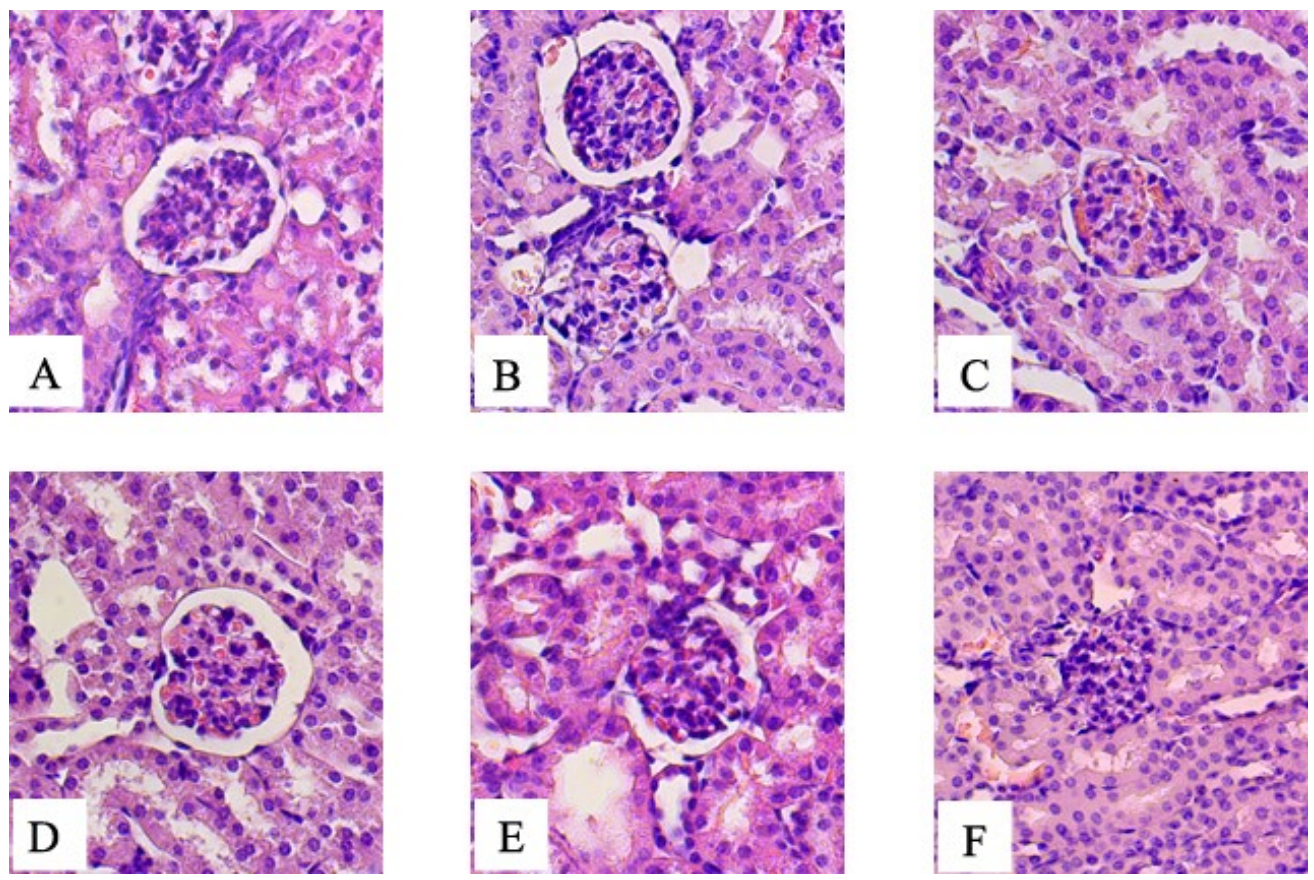

Fig. 2. Histological picture of the kidneys after 2 weeks from the beginning of the experiment. Large magnification: A - control, B - 1st experimental group, C - 2nd experimental group, D - 3rd experimental group, E - 4th experimental group, F - 5th experimental group. Explanations in the text.

There were also no significant differences in the experimental groups both in comparison with the control and with the previous period of the study. Only one animal of the 2nd experimental group showed morphological traits of venous stasis.

Autopsy of mice in the control group revealed a picture identical to intact animals of the previous study period. Autopsy of the animals treated with the new product did not reveal any differences from the control, with the exception of the tendency for a greater body weight of the second experimental group's mice.

1 month from the beginning of the experiment. In the control sections of the kidneys (Fig. 3 ), there were no visible differences in the samples of a similar group of previous periods. Testes preparations (Fig. 4) also showed a typical picture.

The microorganization of the kidneys and testes in most experimental groups' animals also did not reveal potential signs of a negative effect of the studied supplement's components. Leukocyte perivascular infiltrate was identified along the course of some veins in only one 4th experimental group animal in the kidney section at the border of the cortical substance and marrow. 

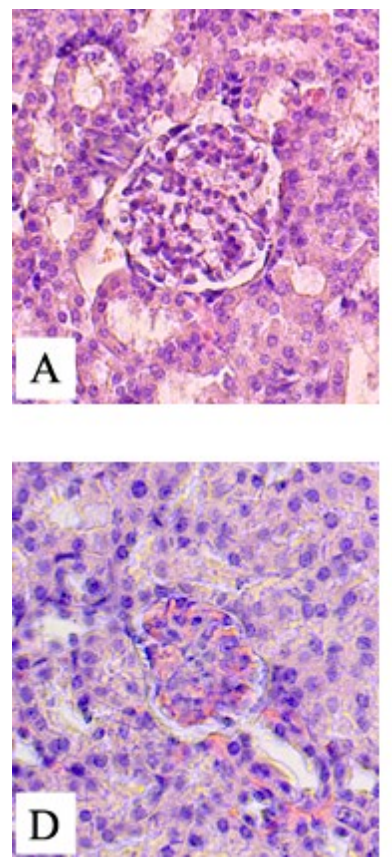
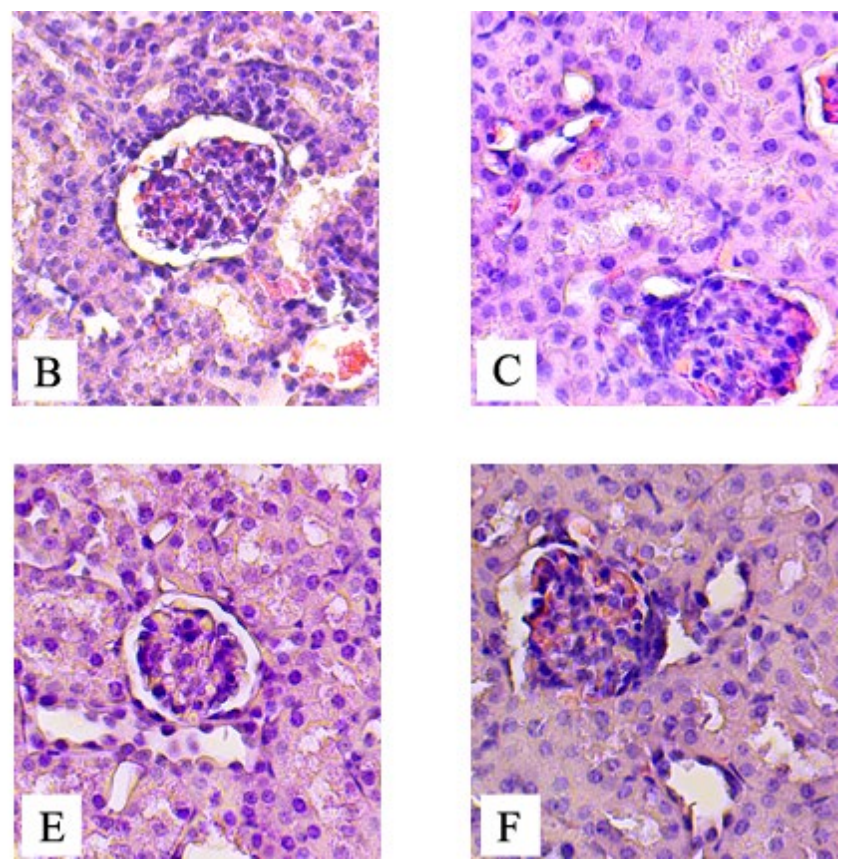

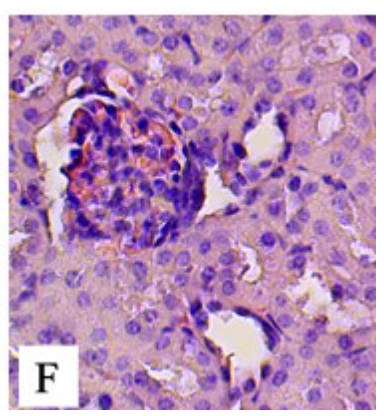

Fig. 3. Histological picture of the kidneys after 1 month from the beginning of the experiment. Large magnification: A - control, B - 1st experimental group, C - 2nd experimental group, D - 3rd experimental group, E - 4th experimental group, F - 5th experimental group. Explanations in the text.
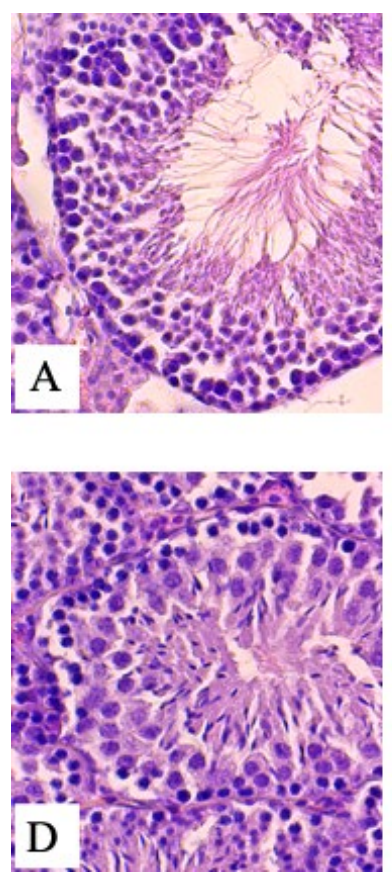
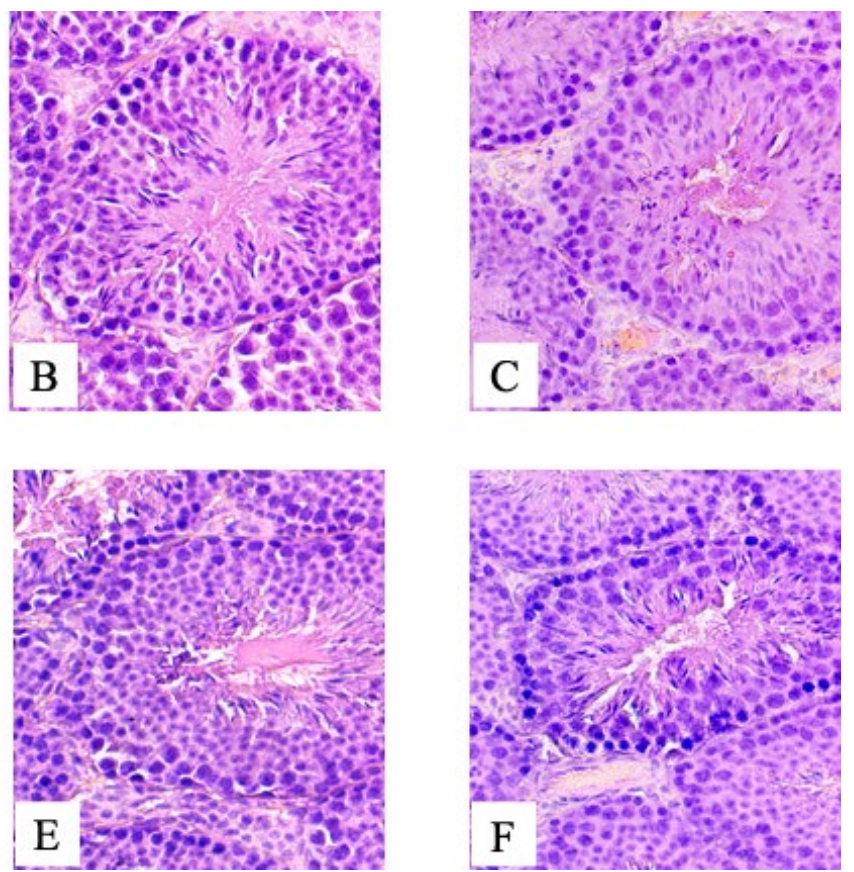

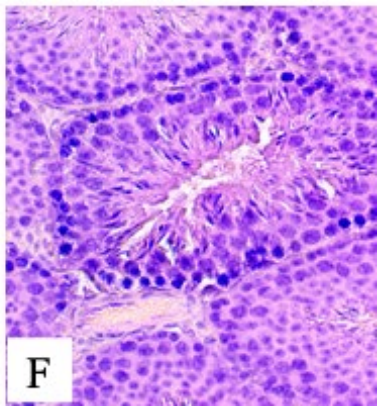

Fig. 4. Histological picture of the testes 1 month after the beginning of the experiment. Large magnification: A - control, B - 1st experimental group, C - 2nd experimental group, D - 3rd experimental group, E - 4th experimental group, F - 5th experimental group. Explanations in the text. 
The pathoanatomical picture in the control group 1 month after the supplements' introduction was the same as in the previous study periods.

The animals of the second experimental group showed a higher body weight but with no traits of obesity. In animals of other experimental groups that received the new product, no differences from the control were found.

\section{Discussion}

The results obtained in combination with previous studies [1, 2, 9] suggest a high bioavailability of the developed supplement's components and the absence of visible negative effects. The applied approach is one of the options for solving problems arising in the development of multicomponent feed additives [4]. The results obtained from the microelements' introduction in the form of chelates are consistent with the data of other authors who received positive effects on the health and productivity of animals [12]. According to other researchers, insufficiently developed or produced supplements or incorrectly calculated dosages can cause a number of negative effects manifested in a violation of the microscopic organization of internal organs. With the excretion of toxic components through the kidneys, infiltrative-dystrophic changes or death of the epithelium of the tubular apparatus with its lifetime desquamation into the lumen of the tubules, a decrease in the number of renal corpuscles, a change in their morphology and other traits of negative influence can develop. These processes can be combined with manifestations of vascular reactions, perivascular infiltration with cells of monocytic and lymphocytic origin, and in later terms, sclerotic changes in the organ. Traits of spermatogenesis depletion may be observed in the testes with a decrease in the spermatozoids' content in the lumen of the convoluted testes' tubules and a decrease in the thickness of their epitheliospermatogenous layer.

The performed histological analysis was aimed primarily at finding such changes, and the study showed the absence of such changes. The revealed single changes in the microorganization of the kidneys and testes are sporadic and are therefore, in the authors' opinion, are consequences of the factors' influence not related to the experiment.

\section{Conclusions}

Thus, the use of the proposed supplement, even in inflated relative to the recommended dosages, for a month does not cause changes in the macroorganization of internal organs, and does not reveal violations of the microscopic picture of the kidneys and testes, which indicates the safety of the excretory and reproductive functions of mice. Single detectable animals with minor foci of inflammatory reaction can be considered as an example of sporadic exposure to pathogenic factors not related to the main experiment.

\section{References}

1. D.S. Berestov, Morphology 157 (2-3), 33 (2020)

2. D.S. Berestov et al., International Veterinary Bulletin, 4, 129-134 (2018)

3. A.V. Shishkin, A.N. Kulikov, M.S. Kulikova et al., Agrarian education and science in the development of animal husbandry: materials of the International Scientific and Practical Conference dedicated to the 70th anniversary of the Honored Worker of Agriculture of the Russian Federation, Honorary Worker of the RF VPO, Laureate of 
the State Prize of the UR, Rector of the Izhevsk SAA, Doctor of Agricultural Sciences, Professor Lyubimov Alexander Ivanovich, 2, 228-230 (2020)

4. A.V. Shishkin, A.N. Kulikov, M.S. Kulikova et al., Integration interactions of young scientists in the development of agricultural science: materials of the National Scientific and Practical Conference of Young Scientists, 2, 448-452 (2020)

5. Y.M. Bao, Journal of Applied Poultry Research, 16(3), 448-455 (2007)

6. V.G. Bhagwat, Veterinary World 14(2), 364-371 (2021)

7. C.R. Dove, Journal of Animal Science 69(5), 1994-2000 (1991)

8. L. Yanhan, Z. Xiaona, Z. Xiao et al., Biological Trace Element Research 171(2), 459467 (2016)

9. D.S. Berestov, M.S. Kulikova, I.S. Ivanov et al., International Journal of Research in Pharmaceutical Sciences 11(3), 4182-4186 (2020)

10. B. Dolińska et al., Biological Trace Element Research 150(1-3), 509-512 (2012)

11. A. Mantovani, Annali dell'Istituto superiore di sanità 42(4), 427-432 (2006)

12. D. Nagalakshmi, Veterinary World 8(9), 1156-1162 (2015)

13. A.J. Pettersen, Comparative Biochemistry and Physiology - Part C: Toxicology \& Pharmacology, 132(1), 53-60 (2002)

14. B. Sandström, British Journal of Nutrition 85, 181-185 (2001)

15. T. Aksu, B. Özsoy, D. S. Aksu et al., Kafkas University Veterinary Medicine Faculty Journal 17(1), 141-146 (2011)

16. T. Belay et al., Journal of Animal Science 96(3), 155-156 (2018) 